\title{
Effect of Torso Non-Homogeneities in the quasi-static inverse problems arising in electrocardiology
}

\author{
BedrEddine Ainseba $^{1}$, Mostafa Bendahmane ${ }^{2}$ and Alejandro Lopez ${ }^{3}$
}

\begin{abstract}
АвSTRACT. In the present paper, an homogeneous and non-homogeneous inverse problem constrained by the stationary problem in electrocardiology representing the heart, lungs surfaces, and torso model is investigated. Our goal is to reconstruct the electrical potentials on the surface of the heart from the information obtained non invasively on the torso surface. The existence and uniqueness of solution for the heart-torso problem and the related inverse problem is assessed, and the primal and dual problems are discretized using a finite element method. We present some preliminary numerical experiments using an efficient implementation of the proposed scheme in homogeneous and non-homogeneous cases. Finally, we demonstrate the effect of the non-homogeneity on the reconstructed epicardial potential and show that the inverse ECG problem cannot be solved by the classical BEM (boundary element method).
\end{abstract}

Mathematics Subject Classification (2010). Primary 35K57, 35M10; Secondary: 35A05.

Key words and phrases. Bioelectric model, weak solution, Homogeneous, non-homogeneous, Direct and Inverse problems, finite element, cardiac electric field.

\section{Introduction}

Cardiovascular disease is the second most common cause of mortality after cancer. Mathematical models of heart are an established approach to diagnosing cardiovascular disease. The obvious difficulty of performing direct measurements in electrocardiology has motivated

\footnotetext{
Received 27 October 2019 - Accepted: 15 December 2019.

C) The Author(s) 2017. This article is published with open access by Sidi Mohamed Ben Abdallah University.

1,2 Institut Mathématiques de Bordeaux, Université de Bordeaux , 351 Cours de la Libération 33400 Talence, France.

e-mail: ${ }^{1}$ bedreddine.ainseba@u-bordeaux.fr

e-mail: ${ }^{2}$ mostafa.bendahmane@u-bordeaux.fr

${ }^{3}$ Universiteitsweg 99, Kamer 0, 3584 CG Utrecht, Holland.

e-mail: ${ }^{3}$ alejandro.lopez@iscpif.fr .
} 
wide interest in the numerical simulation of cardiac models. In 1952, Hodgkin and Huxley [11] introduced the first mathematical model of wave propagation in squid nerve, which was modified later on to describe several phenomena in biology. This led to the first physiological model of cardiac tissue [19] and many others. It is well known [22], that the electrical potential distribution over the body surface (body surface potentials) is the result of the bioelectrical activity within the heart through the intervening tissue to the body surface.

In electrocardiology, computational models of bioelectric phenomenon from the heart have existed for over 40 years. In these models the electric signals in the body produced by the heart can be described as a solution to a quasistatic Poisson equation [22]. The analytic solutions of these equations are not difficult to obtain for simple geometries such as cylinders or spheres. Moreover, the difficulties arise when we consider the complex geometries associated with physiological structures (heart, Lungs and torso). In our work realistic geometries are used to approximate the electrical potential in the heart (inverse problem) and in the torso (direct problem).

The numerical solution of ECG (electrocardiogram) direct and inverse problems has received much attention for many years (and there have been many contributions on this subject) as a means of providing insight into the connection between observable data. The goal of the inverse problem in electrocardiography is the reconstruction of the cardiac electrical potentials from measurements of the potential on the body surface. Here, we mean a mathematical processing of the data obtained on the surface of the body. The main objective of the inverse problem in electrocardiography is to determine, in a noninvasive way, information about the electrophysiological state of the heart, that visual inspection of the electrocardiograph or the body surface mapping cannot provide.

Our paper provides an overview of computational and numerical techniques applied to a class of bioelectric field problems in homogeneous and non-homogeneous cases. Note that Bioelectric field problems are found in a wide variety of biomedical applications that range from single cells [17], to organs [18], up to models that incorporate partial to full human structures [12], [13].

In this paper our direct and inverse problems reduce to boundary value problems for an elliptic equation. We assume that the electric field generated by the heart is quasi-static. This means that at any instant in time the steady-state conditions are in effect [22]. In passing, we want to mention that an other approach to study inverse problem by using a model consisting of a geometric torso model and a model of the electric activation of the heart based upon for example the bidomain model (see for e.g. $[2,3,4,5]$ and $[7,8,9,10]$ ). This approach will be detailed in a forthcoming paper [1].

We assume that the medium surrounding the body (the air) is nonconductive; thus, the normal derivative of the potential vanishes at the boundary of the insulating medium. Moreover, it is assumed that tissues (Thorax, heart, left and right lung) have a Laplaces equation to govern the potential behaviours according to the theory of the Quasi-static Maxwells equations due to low-frequency response of human tissue.

Before we formulate the inverse problem in mathematical context, we need to introduce the forward problem in electrocardiography. The goal of the forward problem is to compute the body surface potentials from the epicardial potentials. As with the inverse problem, we will refer to the forward problem in electrocardiography simply as the forward problem. Note 
that an understanding of the forward problem is a necessary step towards understanding and solving the inverse problem. In the following section, we formulate both the forward and the inverse problems under some general assumptions about the geometry of the heart-torso system.

The remainder of this paper is organized as follows. Section 2 is devoted to the description of our bioelectric model. In Section 3 we prove the well-posedness of the weak solution to our direct problem. The existence of the solution to the inverse problem will be proved in Section 4. Finally in Section 5, we present the numerical experiments on 3D domains for homogeneous and non-homogeneous cases The paper ends with some comments and remarks.

\section{The mathematical model}

The spatial domain of the medium (thorax, heart, left and right lung) for our models is a bounded open subset $\Omega:=\Omega_{T} \cup \bar{\Omega}_{h} \cup \bar{\Omega}_{L} \cup \bar{\Omega}_{R} \subset \mathbb{R}^{3}$ with a piecewise smooth boundary $\partial \Omega$. Herein, $\Omega_{T}, \Omega_{h}, \Omega_{L}$ and $\Omega_{R}$ are the spatial domains of thorax, heart, left and right lung, respectively, with piecewise smooth boundaries $\partial \Omega_{T}, \partial \Omega_{h}, \partial \Omega_{L}$ and $\partial \Omega_{R}$. The quantities of interest are left lung, right lung and the bathing medium electric potentials, $u_{\mathrm{L}}=u_{\mathrm{L}}(x)$, $u_{\mathrm{R}}=u_{\mathrm{R}}(x)$ and $u_{\mathrm{T}}=u_{\mathrm{T}}(x)$ at $x \in \Omega_{L}, x \in \Omega_{R}$ and $x \in \Omega_{T}$. Observe that $\partial \Omega_{T}=\Sigma \cup \partial \Omega_{h} \cup$ $\partial \Omega_{L} \cup \partial \Omega_{R}$, where $\Sigma$ is a thorax surface. Note that ECG signals monitor the electrical activity of the heart from potential measurements at the torso skin surface $\Sigma$. The conductivity of the tissue is represented by scaled tensors $\mathbf{M}_{\mathrm{L}}(x)$ (for the left lung), $\mathbf{M}_{\mathrm{R}}(x)$ (for the right lung) and $\mathbf{M}_{\mathrm{T}}(x)$ (for the bathing medium).

The direct problem consists of solving the following system: : for $k=L, R, T$

$$
\left\{\begin{array}{l}
-\nabla \cdot\left(\mathbf{M}_{\mathrm{k}}(x) \nabla u_{\mathrm{k}}\right)=0, \quad x \in \Omega_{\mathrm{k}}, \\
u_{\mathrm{L}}=u_{\mathrm{T}} \text { and } \mathbf{M}_{\mathrm{L}}(x) \nabla u_{\mathrm{L}} \cdot \mathbf{n}_{\mathrm{L}}=\mathbf{M}_{\mathrm{T}}(x) \nabla u_{\mathrm{T}} \cdot \mathbf{n}_{\mathrm{L}} \text { on } \partial \Omega_{\mathrm{L}}, \\
u_{\mathrm{R}}=u_{\mathrm{T}} \text { and } \mathbf{M}_{\mathrm{R}}(x) \nabla u_{\mathrm{R}} \cdot \mathbf{n}_{\mathrm{R}}=\mathbf{M}_{\mathrm{T}}(x) \nabla u_{\mathrm{T}} \cdot \mathbf{n}_{\mathrm{R}} \text { on } \partial \Omega_{R}, \\
\left(\mathbf{M}_{\mathrm{T}}(x) \nabla u_{\mathrm{T}}\right) \cdot \mathbf{n}_{\mathrm{T}}=0 \text { on } \Sigma, \\
u_{\mathrm{T}}=u_{\mathrm{e}} \text { on } \partial \Omega_{h},
\end{array}\right.
$$

for $u_{\mathrm{e}}$ given on the epicard $\partial \Omega_{h}$. Moreover, the inverse problem consists on solving the following boundary value problem: for $\mathrm{k}=1, \mathrm{r}, \mathrm{t}$

$$
\left\{\begin{array}{l}
-\nabla \cdot\left(\mathbf{M}_{\mathrm{k}}(x) \nabla u_{\mathrm{k}}\right)=0, \quad x \in \Omega_{\mathrm{k}}, \\
u_{\mathrm{L}}=u_{\mathrm{T}} \text { and } \mathbf{M}_{\mathrm{L}}(x) \nabla u_{\mathrm{L}} \cdot \mathbf{n}_{\mathrm{L}}=\mathbf{M}_{\mathrm{T}}(x) \nabla u_{\mathrm{T}} \cdot \mathbf{n}_{\mathrm{L}} \text { on } \partial \Omega_{L}, \\
u_{\mathrm{R}}=u_{\mathrm{T}} \text { and } \mathbf{M}_{\mathrm{R}}(x) \nabla u_{\mathrm{R}} \cdot \mathbf{n}_{\mathrm{R}}=\mathbf{M}_{\mathrm{T}}(x) \nabla u_{\mathrm{T}} \cdot \mathbf{n}_{\mathrm{R}} \text { on } \partial \Omega_{R} \\
\left(\mathbf{M}_{\mathrm{T}}(x) \nabla u_{\mathrm{T}}\right) \cdot \mathbf{n}_{\mathrm{T}}=0 \text { and } u_{\mathrm{T}}=\bar{u} \text { on } \Sigma
\end{array}\right.
$$

for a given $\bar{u}$ on $\Sigma$ and then determining $u_{T}$ on $\partial \Omega_{h}$. Herein, $\mathbf{n}_{\mathrm{k}}$ is the outward unit normals to $\partial \Omega_{\mathrm{k}}$ for $\mathrm{k}=\mathrm{L}, \mathrm{R}, \mathrm{T}$. 


\section{3. solution of the direct problem}

We assume that the matrix function $\mathbf{M}_{\mathrm{j}}, j \in\{\mathrm{L}, \mathrm{R}, \mathrm{T}\}$, is sufficiently smooth so that the following definition of weak solutions make sense. Furthermore, we assume that $\mathbf{M}_{\mathrm{j}} \in L^{\infty}\left(\Omega, \boldsymbol{R}^{3 \times 3}\right)$ and $\mathbf{M}_{\mathrm{j}} \xi \cdot \xi \geqslant C_{M}|\xi|^{2}$ for a.e. $x \in \Omega$, for all $\xi \in \mathbb{R}^{3}, j \in\{\mathrm{e}, \mathrm{i}, \mathrm{s}\}$, and a constant $C_{M}>0$. Before to define the weak solution, we let

$$
\mathbf{M}(x)=\left\{\begin{array}{ll}
\mathbf{M}_{\mathrm{L}}(x) & \text { for } x \in \Omega_{L \prime}, \\
\mathbf{M}_{\mathrm{R}}(x) & \text { for } x \in \Omega_{R}, \\
\mathbf{M}_{\mathrm{T}}(x) & \text { for } x \in \Omega_{T},
\end{array} \text { and } \mathbf{u}(x)= \begin{cases}u_{R}(x) & \text { for } x \in \Omega_{R}, \\
u_{L}(x) & \text { for } x \in \Omega_{L}, \\
u_{T}(x) & \text { for } x \in \Omega_{T} .\end{cases}\right.
$$

For later reference, we now state the definition of a weak solution for our direct problem (2.1):

Definition 3.1. A function $\mathbf{u}$ is a weak solution of the system (2.1) if $\mathbf{u} \in H^{1}(\Omega)$ and $\mathbf{u}=u_{e}$ on $\Sigma$, and the following identity holds:

$$
\int_{\Omega} \mathbf{M}(x) \nabla \mathbf{u} \cdot \nabla \varphi d x=0
$$

for all test function

$$
\varphi=\left\{\begin{array} { l l } 
{ \varphi _ { L } } & { \text { in } \Omega _ { L \prime } } \\
{ \varphi _ { R } } & { \text { for } \Omega _ { R } , } \\
{ \varphi _ { T } } & { \text { for } \Omega _ { T } , }
\end{array} , \in H ^ { 1 } ( \Omega ) \text { and } \left\{\begin{array}{ll}
\varphi_{R}=\varphi_{T} & \text { on } \partial \Omega_{R}, \\
\varphi_{L}=\varphi_{T} & \text { on } \partial \Omega_{L} .
\end{array}\right.\right.
$$

Theorem 3.1. If $\bar{u} \in H^{1 / 2}(\Sigma)$, then the system (2.1) possesses a unique weak solution.

In Theorem 3.1, $H^{1 / 2}(\Sigma)$ is defined as the following

$$
H^{1 / 2}(\Sigma)=\left\{u \in L^{2}(\Sigma): \quad\|u\|_{H^{1 / 2}(\Sigma)}=\left(\sum_{i=1}^{\infty} \alpha_{i}^{1 / 2} u_{i}^{2}\right)^{1 / 2}<\infty\right\} .
$$

Herein, for $i=1, \ldots, \infty, u_{i}$ are the Fourier coefficients of $u$ relative to the eigenfunctions of the Laplace operator $-\Delta_{\Sigma}$ on $\Sigma$, and the corresponding eigenvalues $\alpha_{i}$.

Proof. Existence: First, we definne the following space

$$
\mathcal{H}=\left\{\varphi \in H^{1}(\Omega): \quad \varphi=0 \text { on } \Sigma\right\} .
$$

Next, we let $\mathbf{v} \in H^{1}(\Omega)$ such that $\mathbf{v}=\bar{u}$ on $\Sigma$. Using the trace theorem (see for e.g. [15]), we get

$$
\|\mathbf{v}\|_{H^{1}(\Omega)} \leq\|\bar{u}\|_{H^{1 / 2}(\Sigma)} .
$$

Moreover, according to the classical operator theory (see for e.g. [14]), there exists $w \in \mathcal{H}$ such that

$$
\int_{\Omega} \mathbf{M}(x) \nabla \mathbf{v} \cdot \nabla \varphi d x=-\int_{\Omega} \mathbf{M}(x) \nabla w \cdot \nabla \varphi d x,
$$

for all $\varphi \in \mathcal{H}$. Now we substitute $\mathbf{u}=\mathbf{v}+w$, we obtain

$$
\int_{\Omega} \mathbf{M}(x) \nabla \mathbf{u} \cdot \nabla \varphi d x=\int_{\Omega} \mathbf{M}(x) \nabla(\mathbf{v}+w) \cdot \nabla \varphi d x=0 \text { and } \mathbf{u}=\mathbf{v}+w=\bar{u} \text { on } \Sigma,
$$


for all $\varphi \in \mathcal{H}$.

Uniqueness: According to Definitions 3.1, the following equation hold for all test function $\varphi \in \mathcal{H}$

$$
\int_{\Omega} \mathbf{M}(x) \nabla\left(\mathbf{u}_{1}-\mathbf{u}_{2}\right) \cdot \nabla \varphi d x=0
$$

where $\mathbf{u}_{1}$ and $\mathbf{u}_{2}$ are two weak solutions. We utilize $\varphi=\mathbf{u}_{1}-\mathbf{u}_{2}$ in (3.2) to obtain (recall that $\mathbf{u}_{1}-\mathbf{u}_{2}=0$ on $\Sigma$ )

$$
\int_{\Omega} \mathbf{M}(x) \nabla\left(\mathbf{u}_{1}-\mathbf{u}_{2}\right) \cdot \nabla\left(\mathbf{u}_{1}-\mathbf{u}_{2}\right) d x=0 .
$$

This implies that $\mathbf{u}_{1}-\mathbf{u}_{2}$ is a constant in $\Omega$ and since $\mathbf{u}_{1}-\mathbf{u}_{2}=0$ on $\Sigma$, we deduce that

$$
\mathbf{u}_{1}-\mathbf{u}_{2}=0 \text { in } \Omega \text {. }
$$

This concludes the proof of Theorem 3.1.

\section{Inverse problem and regularization}

In this section, we prove the existence of the solution to the inverse problem.

Note that from Theorem 3.1, we can define the following bounded linear operator:

$$
\Theta: H^{1 / 2}\left(\partial \Omega_{H}\right) \rightarrow H^{1}(\Omega) \text { such that } \Theta\left(u_{e}\right)=\mathbf{u} \text {. }
$$

Observe that by the uniqueness of the solution $\mathbf{u}$ to the direct problem, the operator $\Theta$ is well-defined.

Next we define $\Theta_{H}$ the operator mapping the electrical potential heart surface $u_{e}$ to the electrical potential thorax surface $\bar{u}$

$$
\Theta_{H}: H^{1 / 2}\left(\partial \Omega_{H}\right) \rightarrow H^{1}(\Sigma) \text { such that } \Theta_{H}\left(u_{e}\right)=\bar{u} \text { on } \Sigma \text {. }
$$

Following the Hilbert Uniqueness Method (HUM) developped by Lions [16], we introduce the adjoint operator:

$$
\begin{aligned}
& \Theta_{H}^{*}: H^{1 / 2}(\Sigma) \rightarrow H^{1 / 2}\left(\partial \Omega_{H}\right) \text { such that } \Theta_{H}^{*}(g)=\left(\mathbf{M}_{\mathrm{T}}(x) \nabla p_{\mathrm{T}}\right) \cdot \mathbf{n}_{\mathrm{T}} \\
& \qquad\left\{\begin{array}{l}
-\nabla \cdot\left(\mathbf{M}_{\mathrm{k}}(x) \nabla p_{\mathrm{k}}\right)=0, \quad x \in \Omega_{\mathrm{k}} \\
p_{\mathrm{L}}=p_{\mathrm{T}} \text { and } \mathbf{M}_{\mathrm{L}}(x) \nabla p_{\mathrm{L}} \cdot \mathbf{n}_{\mathrm{L}}=\mathbf{M}_{\mathrm{T}}(x) \nabla p_{\mathrm{T}} \cdot \mathbf{n}_{\mathrm{L}} \text { on } \partial \Omega_{L^{\prime}} \\
p_{\mathrm{R}}=p_{\mathrm{T}} \text { and } \mathbf{M}_{\mathrm{R}}(x) \nabla p_{\mathrm{R}} \cdot \mathbf{n}_{\mathrm{R}}=\mathbf{M}_{\mathrm{T}}(x) \nabla p_{\mathrm{T}} \cdot \mathbf{n}_{\mathrm{R}} \text { on } \partial \Omega_{R}, \\
\left(\mathbf{M}_{\mathrm{T}}(x) \nabla p_{\mathrm{T}}\right) \cdot \mathbf{n}_{\mathrm{T}}=g \text { on } \Sigma, \\
p_{\mathrm{T}}=0 \text { on } \partial \Omega_{h},
\end{array}\right.
\end{aligned}
$$


For $g$ given we define the operator $\Lambda: H^{1 / 2}(\Sigma) \rightarrow H^{1 / 2}(\Sigma)$

$$
\left\{\begin{array}{l}
-\nabla \cdot\left(\mathbf{M}_{\mathrm{k}}(x) \nabla u_{\mathrm{k}}\right)=0, \quad x \in \Omega_{\mathrm{k}} \\
u_{\mathrm{L}}=u_{\mathrm{T}} \text { and } \mathbf{M}_{\mathrm{L}}(x) \nabla u_{\mathrm{L}} \cdot \mathbf{n}_{\mathrm{L}}=\mathbf{M}_{\mathrm{T}}(x) \nabla u_{\mathrm{T}} \cdot \mathbf{n}_{\mathrm{L}} \text { on } \partial \Omega_{L}, \\
u_{\mathrm{R}}=u_{\mathrm{T}} \text { and } \mathbf{M}_{\mathrm{R}}(x) \nabla u_{\mathrm{R}} \cdot \mathbf{n}_{\mathrm{R}}=\mathbf{M}_{\mathrm{T}}(x) \nabla u_{\mathrm{T}} \cdot \mathbf{n}_{\mathrm{R}} \text { on } \partial \Omega_{R}, \\
\left(\mathbf{M}_{\mathrm{T}}(x) \nabla u_{\mathrm{T}}\right) \cdot \mathbf{n}_{\mathrm{T}}=0 \text { on } \Sigma, \\
u_{\mathrm{T}}=\left(\mathbf{M}_{\mathrm{T}}(x) \nabla p_{\mathrm{T}}\right) \cdot \mathbf{n}_{\mathrm{T}} \text { on } \partial \Omega_{h \prime}
\end{array}\right.
$$

$$
\Lambda(g)=u_{\mathrm{T}} \text { on } \Sigma \text {. }
$$

Multiplying the first equation in 4.2 by $p_{\mathrm{k}}$ solution of 4.1 , integrating over $\Omega_{\mathrm{k}}$, and summing over $k$ one gets:

$$
\int_{\Sigma} u_{\mathrm{T}}(x) g(x) d x=\int_{\partial \Omega_{h}}\left(\mathbf{M}_{\mathrm{T}}(x) \nabla p_{\mathrm{T}} \cdot \mathbf{n}_{\mathrm{T}}\right)^{2} d x
$$

Thus the semi-norm induced by the operator $\Lambda$

$$
<\Lambda g, g>=\int_{\partial \Omega_{h}}\left(\mathbf{M}_{\mathrm{T}}(x) \nabla p_{\mathrm{T}} \cdot \mathbf{n}_{\mathrm{T}}\right)^{2} d x
$$

is in fact a norm (Note that $\Lambda=\Theta_{H} \Theta_{H}^{*}$ ).

We can now introduce a Hilbert space $G$ as the completion of $H^{1 / 2}(\Sigma)$ with respect to the semi-norm induced by $\Lambda$. Then if $\bar{u} \in G^{\prime}$, there exist a unique $g \in G$ s.t. $\Lambda(g)=\bar{u}$, and the inverse problem admits a unique solution $\left(\Lambda\right.$ is an isomorphism from $G$ to its dual space $\left.G^{\prime}\right)$. Since $G^{\prime}$ is an unknown space it's difficult to deal with data in this space. Thus we choose to work in more common spaces for the inverse problem. Namely data in $H^{1 / 2}(\Sigma)$ or in $L^{2}(\Sigma)$.

In the following, we show the ill-posedness of the inverse problem by showing that the operator $\Theta_{H}$ is compact and then the inverse operator $\Theta_{H}^{-1}$ is an unbounded operator. The following theorem is a consequence of the compactness of the operator $\Theta_{H}$ (see [16] for e.g.):

Theorem 1. The inverse problem (2.2) is ill-posed.

Since the inverse problem is ill-posed, the inverse problem (11) is usually solved by regularization methods. We introduce the regularized cost function:

$$
J_{\lambda}\left(u_{e}\right)=\frac{1}{2}\left\|u_{\mathrm{T}}-\bar{u}\right\|_{L^{2}(\Sigma)}^{2}+\frac{\lambda}{2}\left\|u_{e}\right\|_{L^{2}\left(\partial \Omega_{h}\right)}^{2}
$$

and look for its minimum over $u_{e}$ (recall that in the direct problem (2.1), $u_{\mathrm{T}}=u_{e}$ on $\partial \Omega_{\mathrm{h}}$ ). Using the Lagrangian method we can show that the optimality system is given by:

$$
\left\{\begin{array}{l}
-\nabla \cdot\left(\mathbf{M}_{\mathrm{k}}(x) \nabla p_{\mathrm{k}}\right)=0, \quad x \in \Omega_{\mathrm{k}}, \\
p_{\mathrm{L}}=p_{\mathrm{T}} \text { and } \mathbf{M}_{\mathrm{L}}(x) \nabla p_{\mathrm{L}} \cdot \mathbf{n}_{\mathrm{L}}=\mathbf{M}_{\mathrm{T}}(x) \nabla p_{\mathrm{T}} \cdot \mathbf{n}_{\mathrm{L}} \text { on } \partial \Omega_{\mathrm{L}}, \\
p_{\mathrm{R}}=p_{\mathrm{T}} \text { and } \mathbf{M}_{\mathrm{R}}(x) \nabla p_{\mathrm{R}} \cdot \mathbf{n}_{\mathrm{R}}=\mathbf{M}_{\mathrm{T}}(x) \nabla p_{\mathrm{T}} \cdot \mathbf{n}_{\mathrm{R}} \text { on } \partial \Omega_{R}, \\
\left(\mathbf{M}_{\mathrm{T}}(x) \nabla p_{\mathrm{T}}\right) \cdot \mathbf{n}_{\mathrm{T}}=u_{\mathrm{T}}-\bar{u} \text { on } \Sigma, \\
p_{\mathrm{T}}=0 \text { on } \partial \Omega_{h},
\end{array}\right.
$$




$$
\left\{\begin{array}{l}
-\nabla \cdot\left(\mathbf{M}_{\mathrm{k}}(x) \nabla u_{\mathrm{k}}\right)=0, \quad x \in \Omega_{\mathrm{k}}, \\
u_{\mathrm{L}}=u_{\mathrm{T}} \text { and } \mathbf{M}_{\mathrm{L}}(x) \nabla u_{\mathrm{L}} \cdot \mathbf{n}_{\mathrm{L}}=\mathbf{M}_{\mathrm{T}}(x) \nabla u_{\mathrm{T}} \cdot \mathbf{n}_{\mathrm{L}} \text { on } \partial \Omega_{L}, \\
u_{\mathrm{R}}=u_{\mathrm{T}} \text { and } \mathbf{M}_{\mathrm{R}}(x) \nabla u_{\mathrm{R}} \cdot \mathbf{n}_{\mathrm{R}}=\mathbf{M}_{\mathrm{T}}(x) \nabla u_{\mathrm{T}} \cdot \mathbf{n}_{\mathrm{R}} \text { on } \partial \Omega_{R}, \\
\left(\mathbf{M}_{\mathrm{T}}(x) \nabla u_{\mathrm{T}}\right) \cdot \mathbf{n}_{\mathrm{T}}=0 \text { on } \Sigma, \\
u_{\mathrm{T}}=\frac{1}{\lambda}\left(\mathbf{M}_{\mathrm{T}}(x) \nabla p_{\mathrm{T}}\right) \cdot \mathbf{n}_{\mathrm{T}} \text { on } \partial \Omega_{h},
\end{array}\right.
$$

Note that the solution of this optimality system is equivalent to

$$
u_{e}^{\lambda}=\left(\Theta_{H}^{*} \Theta_{H}+\lambda I\right)^{-1} \Theta_{H}^{*} \bar{u},
$$

which is the Tikhonov regularization introduced originally in [23].

\section{Numerical results}

In this section, we reconstruct the electric potential in the heart surface from body surface maps. We will show the effect of torso inhomogeneities in the reconstruction of epicardial potentials. In our numerical results, three types of realistic torso models were used in calculating the inverse electric potential in the heart, and the effects of torso inhomogeneities using the finite element method approximation. The first model is an homogeneous model with an average conductivity for the whole torso. The second model is a non-homogeneous model with different conductivity parameters for lungs, and heart ventricles cavities filled with blood. In the third model we use the geometry from the non-homogeneous model, but with an average conductivity for the whole torso. This means that in this case we use the mesh of the homogeneous case by adding the positions of lungs and heart ventricles cavities. Using real measured data, our study shows that the difference between the homogeneous and non-homogeneous models depends not only of the conductivity parameters, but also on the geometry used.

5.1. Construction of the numerical scheme. For numerical simulation, we consider a finite element approximation. For that we suppose that $\bar{\Omega}$ is a polyhedron. This allows us to cover the sets $\bar{\Omega}$, respectively $\partial \Omega$, with polygonal 3-dimensional. In this way we construct a triangulation $\mathcal{T}_{h}$ of $\Omega$ which satisfies the usual assumptions [6] (page 8) and we introduce the finite element space $V_{h}$ approximating $H^{1}(\Omega)$ by piecewise trilinear continuous functions on $\mathcal{T}_{h}$, for wich $\varphi_{h}$ is a basis. Let $\mathbf{M}_{j}^{h}$ be the finite element interpolants of $\mathbf{M}_{j}$ for $j=L, R, T$,

$$
\mathbf{M}^{h}(x)=\left\{\begin{array}{ll}
\mathbf{M}_{\mathrm{L}}^{h}(x) & \text { for } x \in \Omega_{L}, \\
\mathbf{M}_{\mathrm{R}}^{h}(x) & \text { for } x \in \Omega_{R}, \\
\mathbf{M}_{\mathrm{T}}^{h}(x) & \text { for } x \in \Omega_{T},
\end{array} \text { and } \mathbf{u}_{h}(x)= \begin{cases}u_{R}^{h}(x) & \text { for } x \in \Omega_{R}, \\
u_{L}^{h}(x) & \text { for } x \in \Omega_{L}, \\
u_{T}^{h}(x) & \text { for } x \in \Omega_{T}, \\
u_{e}^{h}(x) & \text { for } x \in \Sigma,\end{cases}\right.
$$

The classic Galerkin formulation of the problem: Find $\mathbf{u}_{h}$ such that

$$
\int_{\Omega} \mathbf{M}^{h}(x) \nabla \mathbf{u}_{h} \cdot \nabla \varphi_{h} d x=0
$$


for all test function

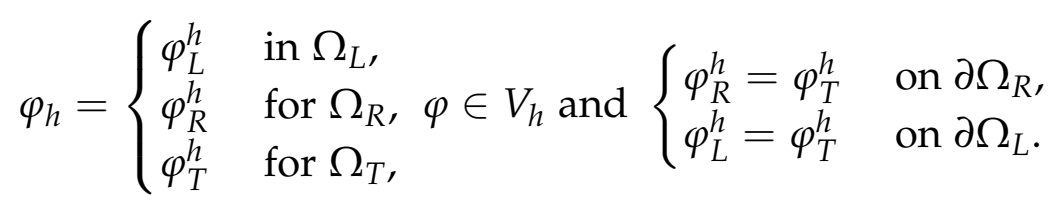

5.2. Construction of the Transfer Matrix. Note that the Inverse problem consists in three steps:

- Create the mesh for $\Omega$ (the mesh is created using the *.Msh Files).

- Calculate the Transfer Matrix K (this matrix describes the relation between the cardiac sources and the measurement signal on the body surface).

- Solve the system $K U=F$, where $U$ is the source vector and $F$ is the measurement vector.

To calculate the Transfer Matrix we need to calculate the Stiffness Matrix. The following is the Stiffness Matrix which (it is obtained from the equation (5.1)) we will divide in the following submatrixes:

$$
\left[\begin{array}{ccc}
A_{h h} & A_{h v} & A_{h t} \\
A_{v h} & A_{v v} & A_{v t} \\
A_{t h} & A_{t v} & A_{t t}
\end{array}\right]
$$

where $h, v, t$ denote nodal indexes that lie on Heart, Volume (Lungs) and Torso respectively. The submatrixes $A_{h t}$ and $A_{t h}$ are zero because we consider that there is no overlapping of the sub-domains. Observe that from (5.1), we get:

$$
\left[\begin{array}{cc}
A_{v v} & A_{v t} \\
A_{t v} & A_{t t}
\end{array}\right]\left[\begin{array}{l}
u_{v} \\
u_{t}
\end{array}\right]=\left[\begin{array}{l}
-A_{v h} \\
-A_{t h}
\end{array}\right] u_{h},
$$

where $u_{t}, u_{h}$ and $u_{v}$ are respectively the vector of electrical potentials at the nodes on the torso surface, the vector of electrical potentials at the nodes located on the epicardium and the electrical potentials at the rest of the nodes in the volume conductor. Note that from (5.2), we deduce easily

$$
u_{v}=-A_{v v}^{-1}\left(A_{v h} u_{h}+A v t u_{t}\right),
$$

and the Transfer Matrix will have the following form:

$$
K=\left(A_{t t}-A_{t v} A_{v v}^{-1} A_{v t}\right)^{-1} A_{t v} A_{v v}^{-1} A_{v h} .
$$

Since the inverse problem is an ill-posed problem a regularization technique it is necessary. The regularization technique used in this study is a global Tikhonov scheme. For this global scheme, the solution $u_{0}$, can be estimated by minimizing a generalized form of the discretized Tikhonov functional:

$$
\min \left(\left\|K u_{h}-u_{t}\right\|^{2}+\lambda\left\|C\left(u_{h}-u_{h}^{\prime}\right)\right\|^{2}\right), \quad \lambda>0,
$$

where $C$ is a constrained matrix (the identity matrix) and $u_{h}^{\prime}$ is the priori information. In our numerical simulation we use $u_{h}^{\prime}=0$. 
5.3. Regularization Parameter. To find the optimal value of regularization parameter, we use the L-Curve Method. This method (L-Curve) is a parametric plot of $\left(\log _{10}\left(\left\|A x_{\lambda}-b\right\|_{2}\right.\right.$ ), $\left.\log _{10}\left(\left\|x_{\lambda}\right\|_{2}\right)\right)$ for different values of the regularization parameter $\lambda$. The optimal value of Lambda for Thikonov-0 regularization can be obtained by the maximum value of the curvature given by the following formula:

$$
\kappa(\lambda)=\frac{\rho^{\prime \prime} * \eta^{\prime}-\rho^{\prime} * \eta^{\prime \prime}}{\left(\left(\rho^{\prime}\right)^{2}+\left(\eta^{\prime}\right)^{2}\right)^{3 / 2}}
$$

where:

$$
\begin{gathered}
\rho=\log _{10}\left(\left\|A x_{\lambda}-b\right\|_{2}\right) \\
\eta=\log _{10}\left(\left\|x_{\lambda}\right\|_{2}\right)
\end{gathered}
$$

An example of 500 points L-curve, for data measured in the thorax, can be seen in Figure 1, for the values between 0.1 to 0.00001 of $\lambda$. In our study the value $\lambda=0.001$ was used, based on the result for different data in the thorax.

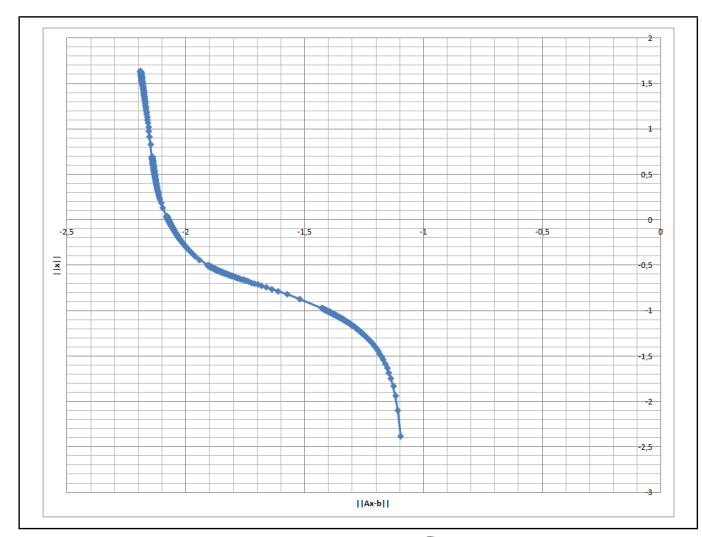

FigURE 1. L-Curve.

5.4. Numerical Results for Inverse Problem. To make the test we used the data from ECGSIM. ECGSIM is an interactive simulation program that enables to study the relationship between the electric activity of the heart and the resulting potentials on the thorax (see for e.g. [20,21]). We would like to mention here that ECGSIM does not solve the inverse problem.

From the ECGSIM we take the surfacic geometry, the measured ECG and the the simulated ECG with the initial parameter values. Having the surfacic geometry (from ECGSIM), we generate the volumetric mesh by using a three-dimensional finite element mesh generator Gmesh. With the volumetric mesh we generate the Transfer Matrix for the both cases, homogeneous (heart and thorax only) and non homogeneous (heart, thorax and lungs).

In our simulation we use a measured ECG of 64 nodes on the body surface of a normal male ( 25 years $100 \mathrm{~kg}$ ) and then interpolated to the rest of the thorax for 1000 samples nodes. The values in the heart are calculated using as source model the equivalent double layer (EDL). The map of the voltage in the thorax is given in Figure 2. 


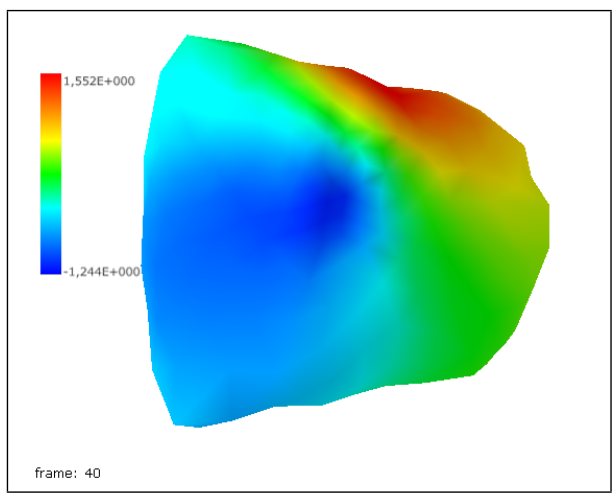

FIGURE 2. Real data on thorax
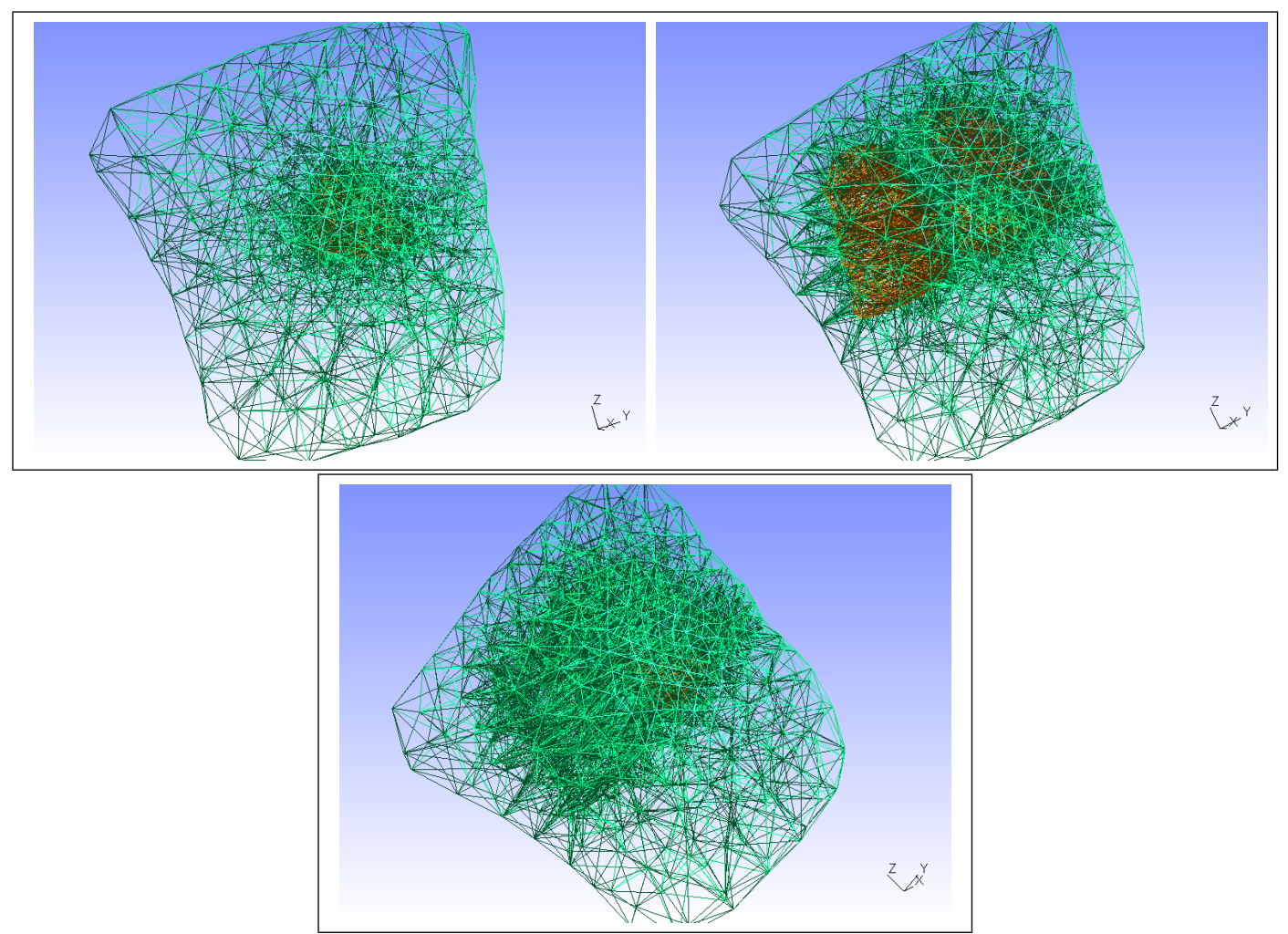

Figure 3. Homogeneous Torso (top left), Non-Homogeneous Torso (top right) and Homogeneous Torso including the geometries of lungs (bottom)

Three torso models were used (see Figure 3). The first one is a homogeneous torso model with conductivity value $\sigma=2.4$ (see Figure 3 top left). The second torso model is a nonhomogeneous torso model with conductivity values, for lungs $\left(\sigma_{l} u n g s=0.4\right)$, for heart ventricles cavities $\left(\sigma_{c} a v=6.0\right)$, and for the rest $\left(\sigma_{r} e s t=2.4\right)$, see Figure 3, top right. The third torso model is homogeneous torso model with conductivity value $\sigma=2.4$, but using the same geometry from the second model (see Figure 3, bottom). 
Test1:

Using real data in the thorax the inverse solution was calculated for the three torso models

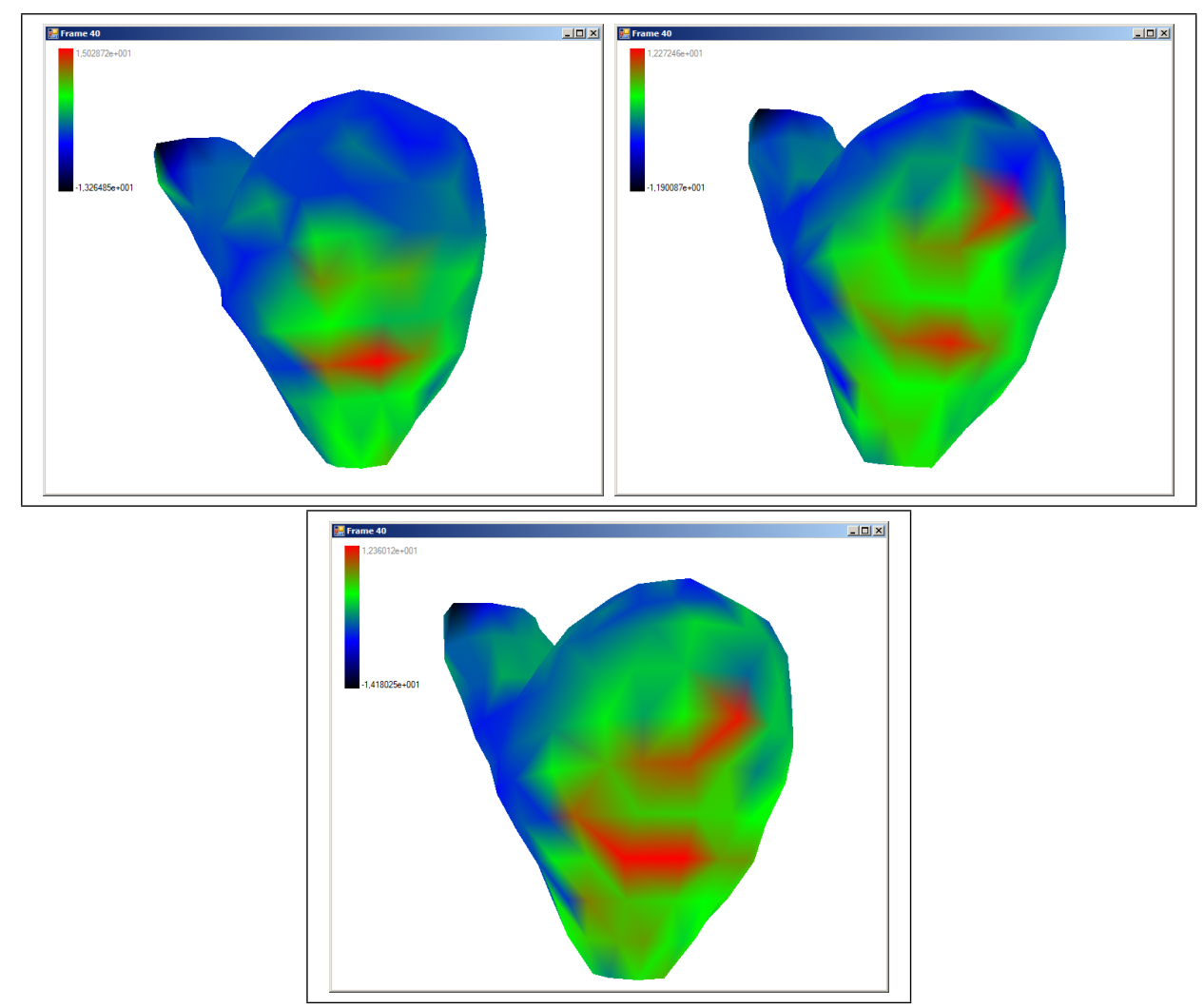

FIGURE 4. Inverse solution for $t=40 \mathrm{~ms}$ in Homogeneous Torso (Figure 4 , left), in Non-Homogeneous Torso (Figure 4, right) and in Homogeneous Torso including the geometries of lungs (Figure 4 , bottom)

in Figure 3. At time $t=40 \mathrm{~ms}$, we observe the global difference between the inverse problem for the first model (Figure 4, top left)) and for the second model (Figure 4, right)). Moreover the inverse problem for the second model (Figure 4, top left) and the third model (Figure 4, bottom) are very similar. In our first test the comparison in some nodes (nodes 202 and 207) between the first and the second model, and between the second and the third model are given in Figures 5 and 6.

\section{Test2:}

In the second test, the data on the heart was generated by a synthetic data generated by solving the bidomain equations. The original data on the heart is shown in the Figure 7 at time $t=200 \mathrm{~ms}$. In our numerical simulation we use the value of regularization $\lambda=$ 0.001. Using the calculated values in the thorax, the inverse solution was calculated for the three torso models: homogeneous, non-homogeneous, and non-homogeneous geometry, with homogeneous conductivity (See Figures 8). 


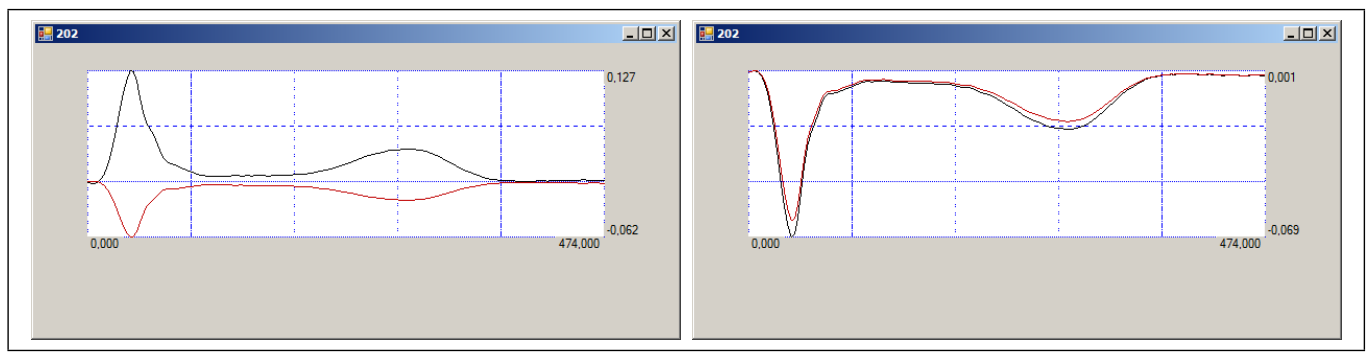

FiguRE 5. Comparison between the first and the second model (left), and between, the second and the third model in the node 202

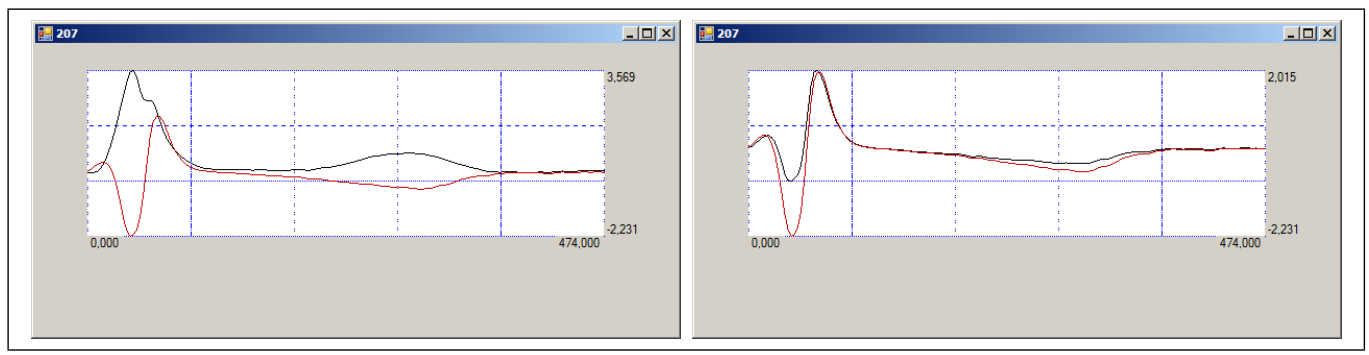

FiguRE 6. Comparison between the first and the second model (left), and between, the second and the third model in the node 207

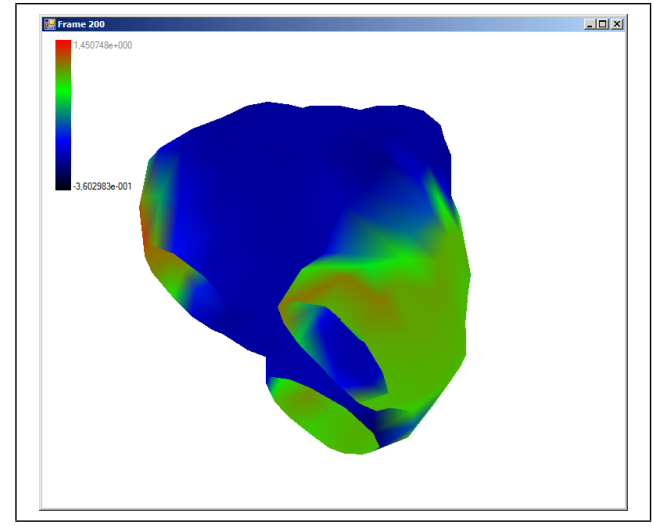

FigURE 7. Real data on the heart for $\mathrm{t}=200 \mathrm{~ms}$.

\section{Test3:}

In the third test, we use the data generated in the thorax from the electrical activity in the heart of Figure 7. Then, the value of regularization was modified to $\lambda=0.0$, and the inverse solution was calculated for the three torso models: homogeneous, non-homogeneous, and non-homogeneous geometry, with homogeneous conductivity (see Figure 9).

We observe that in the non-homogeneous case, the solution on the heart's surface, resembles to the case when we use $\lambda=0.001$. This is because, the data in the thorax was generated using the non-homogeneous transfer matrix. Moreover, if the data in the thorax was generated using the homogeneous transfer matrix, and the calculated the inverse solution in the heart's 


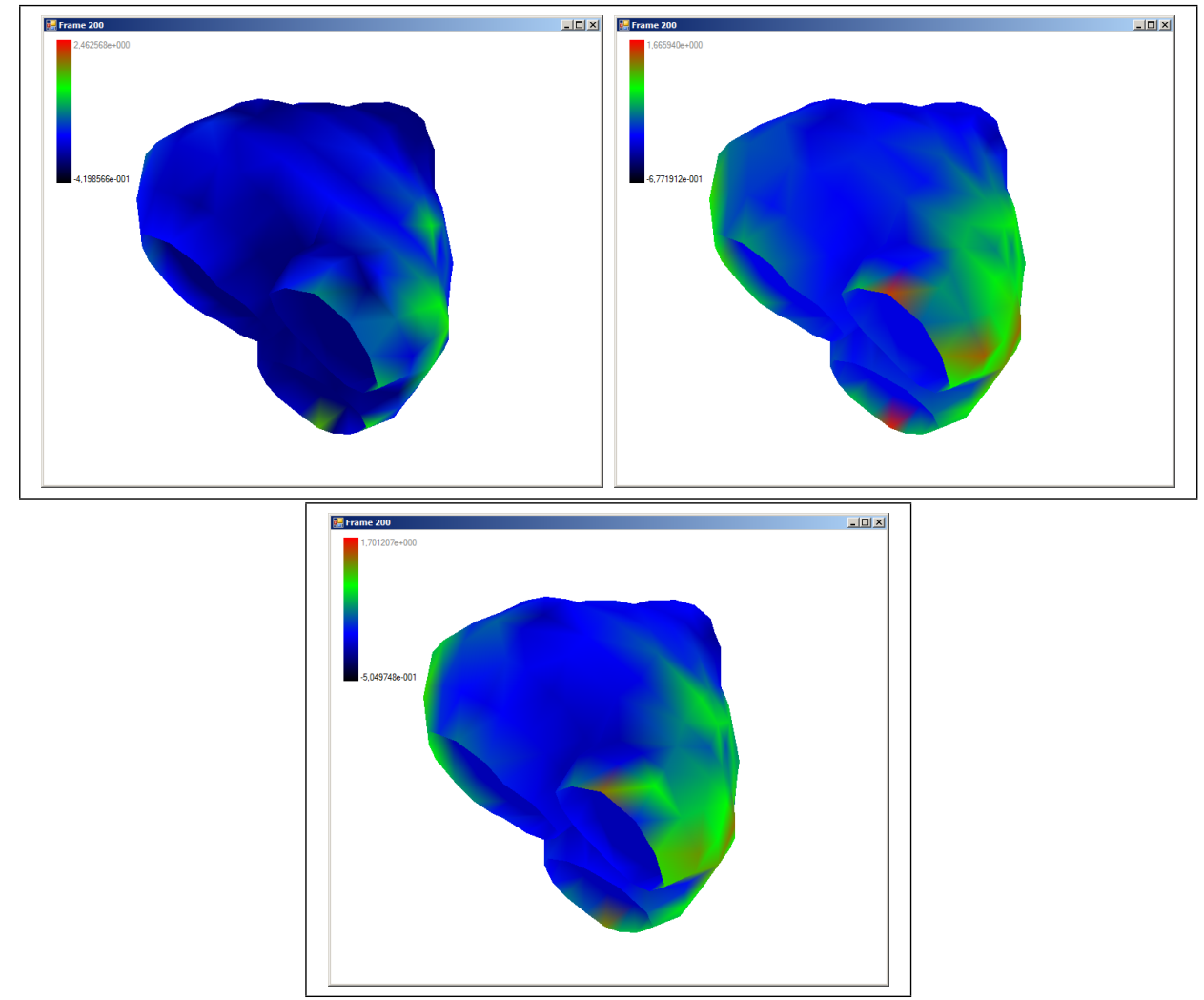

FiguRE 8. Inverse solution on the heart at time $t=40 \mathrm{~ms}$ for Homogeneous Torso (left), for Non-Homogeneous Torso (right) and in Homogeneous Torso including the geometries of lungs (bottom)

surface, for the non-homogeneous torso model with $\lambda=0.0$, then we get the result obtained in Figure 10.

\section{Test4:}

In our last test, we use the data generated in the thorax from the electrical activity in the heart of Figure 7. Then, the value of regularization was modified to $\lambda=1.0$, and the inverse solution was calculated for the three torso models: homogeneous, non-homogeneous, and nonhomogeneous geometry, with homogeneous conductivity (see Figure 11). We observe that in this case we obtain the same inverse solution significantly different to the real data on the heart.

\section{Conclusions}

As mentioned before the problem of inverse electrocardiology is an ill-posed problem. This means that a small perturbation can change the response in the heart. This is the reason why it is necessary to make the regularization. In this test we made a comparison between the 


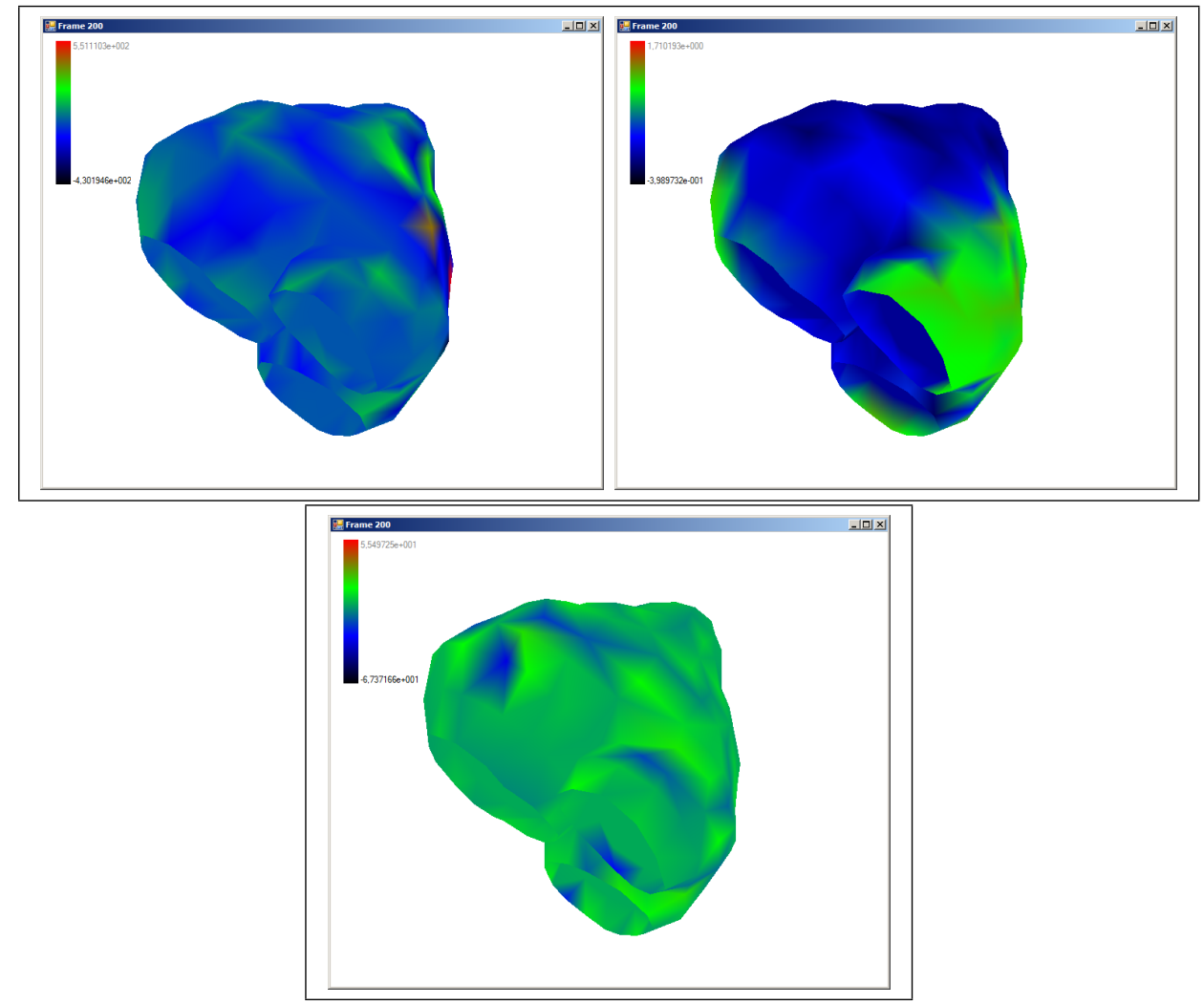

Figure 9. On the heart at time $t=200 \mathrm{~ms}$, Homogeneous, Non-Homegeneous and Non-Homogeneous geometry with homgeneous condutivity solution

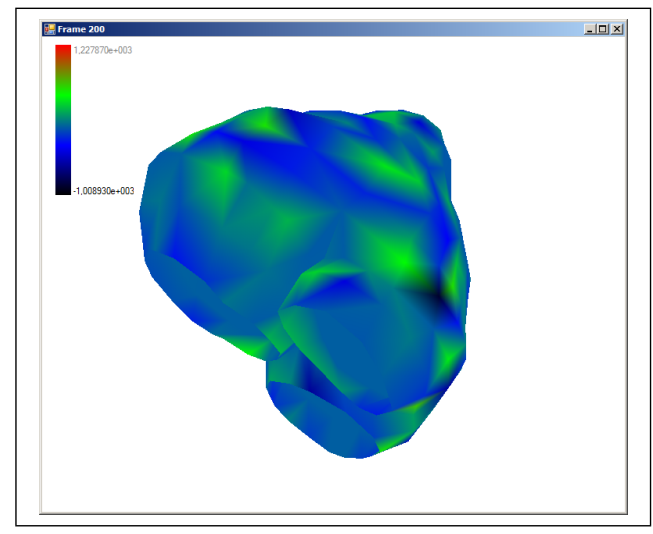

FigURE 10. Non-Homogeneous Solution on the heart for $\mathrm{t}=200 \mathrm{~ms}$.

calculated surface voltage nodal values with different values for the tikhonov regularization parameter $\lambda$, and the double layer EDL surface source model used by the ECGSIM software.

One of the points in the inverse problem is the choice of the regularization parameter $\lambda$, as seen in the simulations an small change in $\lambda$ changes drastically the result in the heart surface. To choose the value of $\lambda$, a comparison has to be made with a source model: in this 


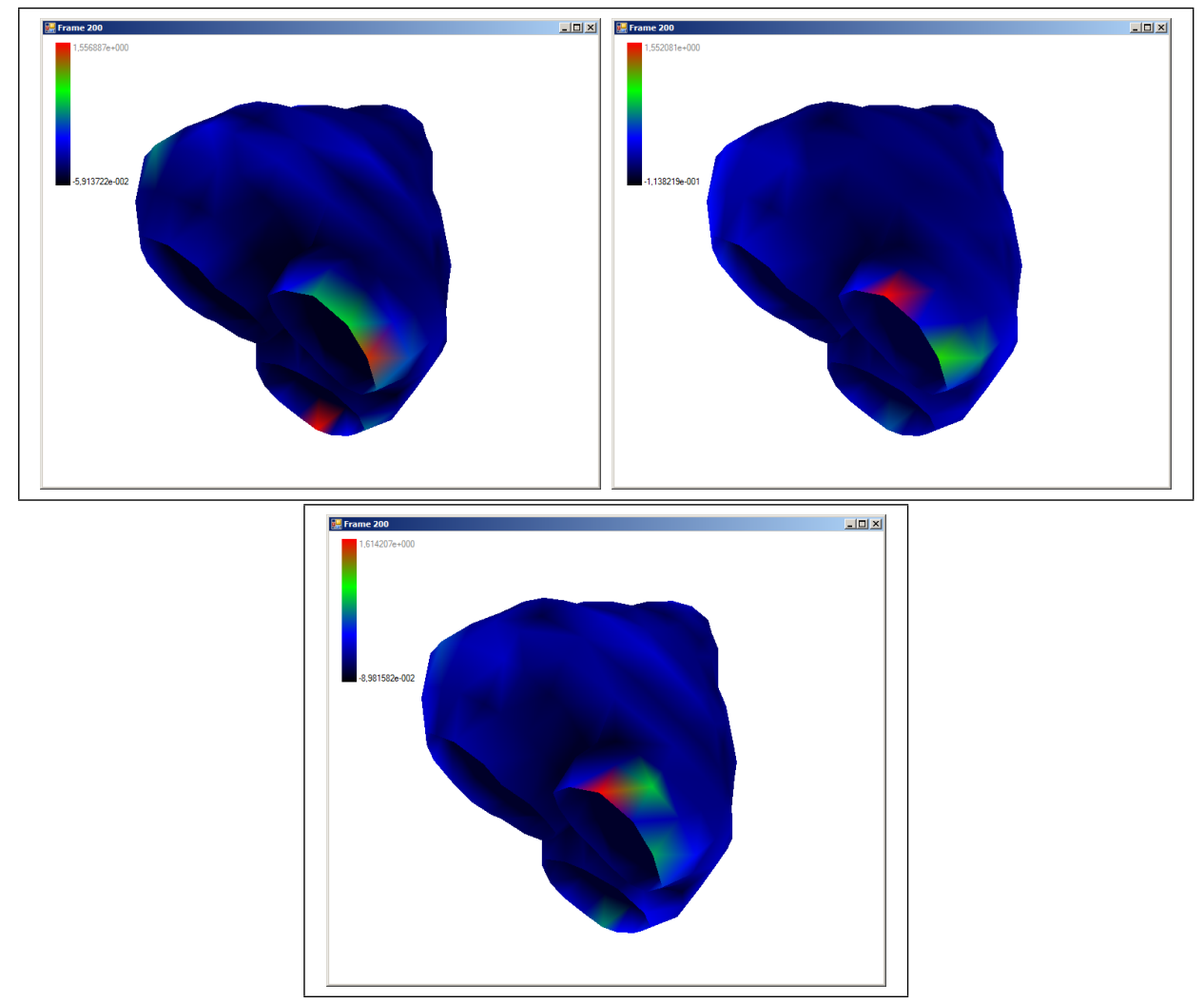

Figure 11. On the heart at time $t=200 \mathrm{~ms}$, Homogeneous, Non-Homegeneous and Non-Homogeneous geometry with homgeneous condutivity solution

case the model was the EDL source model. Once we had the value of $\lambda$, we made the test for the homogeneous and non homogeneous case. From the numerical tests we conclude that the homogeneous and non homogeneous case is the same for the direct problem, but the results in the surface of the heart are different for the inverse problem. We can see that differences depend of the geometry: the differences are presented in the nodes closest to the back, where the consideration of the different conductivity of the lungs affects the more.

Other problem to consider is that the calculation of the transfer matrix, which changes from person to person, because of the geometry. During the calculation of this matrix there is a computational problem because it is necessary as one of the steps to make the calculation of the inverse from the sub-matrix $A_{v v}$, which depends on the density of the geometry and has the size of the nodes in the volume in between the surface of the heart and the surface of the thorax.

\section{References}

[1] B.E. Ainseba, M. Bendahmane and A. Lopez. Analysis of an inverse problem for the bidomain model of cardiac tissue. Preprint.

[2] M. Bendahmane, R. Bürger and R. Ruiz. A finite volume scheme for cardiac propagation in media with isotropic conductivities. Mathematics and Computers in Simulation, (80):1821-1840, 2010. 
[3] M. Bendahmane, R. Bürger and R. Ruiz. A multiresolution space-time adaptive scheme for the bidomain model in electrocardiology. Num. Met. for Part. Diff. Eq., 26, 1377-1404, 2010.

[4] M. Bendahmane and K.H. Karlsen, Analysis of a class of degenerate reaction-diffusion systems and the bidomain model of cardiac tissue, Netw. Heterog. Media 1 (2006) 185-218.

[5] M. Bendahmane and K.H. Karlsen. Finite volume methods for degenerate reaction-diffusion systems modeling the Cardiac electric field. Applied Numerical Mathematics, (59)9, 2266-2284, 2009.

[6] P.G. Ciarlet. The finite element method for elliptic problems. North-Holland Publishing Co, Amsterdam, 1978 Studies in Mathematics and its Applications, Vol 4.

[7] P. Colli Franzone, P. Deuflhard, B. Erdmann, J. Lang, L.F. Pavarino, Adaptivity in space and time for reactiondiffusion systems in electro-cardiology, SIAM J. Sci. Comput. 28 (2006) 942-962.

[8] P. Colli Franzone, L.F. Pavarino, A parallel solver for reaction-diffusion systems in computational electrocardiology, Math. Models Meth. Appl. Sci. 14 (2004) 883-911.

[9] P. Colli Franzone, L.F. Pavarino, B. Taccardi, Simulating patterns of excitation, repolarization and action potential duration with cardiac Bidomain and Monodomain models, Math. Biosci., 197 (2005), 35-66.

[10] P. Colli Franzone, G. Savaré, Degenerate evolution systems modeling the cardiac electric field at microand macroscopic level, in: A. Lorenzi, B. Ruf (Eds.), Evolution equations, semigroups and functional analysis, Birkhäuser, Basel, 2002, pp. 49-78.

[11] A.L. Hodgkin, A.F. Huxley, A quantitative description of membrane current and its application to conduction and excitation in nerve, J. Physiol. 117 (1952) 500-544.

[12] C.R. Johnson, R.S. MacLeod and P.R. Ershler. A computer model for the study of electrical current flow in the human thorax. Computers in Biology and Medicine, 1992;22:305323.

[13] C.R. Johnson, R.S. MacLeod and M.A. Matheson. Computer simulations reveal complexity of electrical activity in the human thorax. Comp. in Physics, 1992;6:230237.

[14] O. A. Ladyzhenskaya and N. N. Ural'tseva. Linear and quasilinear elliptic equations. Academic Press, New York, 1968.

[15] J.-L. Lions. Quelques méthodes de résolution des problèmes aux limites non linéaires. Dunod, 1969.

[16] J.-L Lions. Contrôlabilité Exacte, Perturbation et Stabilisation de Systèmes Distribués. Masson, 1986.

[17] C.E. Miller and C.S. Henriquez. Finite element analysis of bioelectric phenomena. Crit. Rev. in Biomed. Eng., 1990;18:181205.

[18] J. Nenonen, H.M. Rajala and T. Katilia. Biomagnetic Localization and 3D Modelling. Helsinki University of Technology, Espoo, Finland, 1992, Report TKK-F-A689.

[19] D. Noble, A modification of the Hodgkin-Huxley equations applicable to Purkinje fibre action and pacemaker potentials, J. Physiol. 160 (1962) 317-352.

[20] A. van Oosterom and T. Oostendorp, ECGSIM; an interactive tool for studying the genesis of QRST waveforms. Heart 90 (2004), 165-168.

[21] A. van Oosterom, Interactive simulation of the QRS wave forms. In: Images of the Twenty-first Century. Proc. of the Annual Internat. Conf. of the IEEE Eng. in Med. and Biol. Soc. edt: Y. Kim, F.A. Spelman IEEE Publishing Services, New York, 1989, 183-184.

[22] R. Plonsey, Bioelectric Phenomena, McGraw-Hill, New York, 1969.

[23] A. N. Tikhonov. Regularization of incorrectly posed problems. Soviet Math. Dokl., 4:16241627, 1963. 